\title{
Robinow Syndrome and Brachydactyly: An Interplay of High-Throughput Sequencing and Deep Phenotyping in a Kindred
}

\author{
Ranjana Mishra ${ }^{a}$ Vibha Jain ${ }^{a}$ Deepti Gupta ${ }^{a}$ Renu Saxena ${ }^{a}$ \\ Samarth Kulshreshtha ${ }^{a}$ Vedam L. Ramprasad ${ }^{b}$ Ishwar C. Verma ${ }^{a}$ \\ Ratna Dua Puria \\ ${ }^{a}$ Institute of Medical Genetics and Genomics, Sir Ganga Ram Hospital, New Delhi, India; ${ }^{b}$ Medgenome Labs, \\ Bangalore, India
}

\section{Established Facts}

- Robinow syndrome is a skeletal syndrome with fetal facies and genital anomalies.

- The utility of whole-exome sequencing (WES) in unrecognizable or intricate phenotypes is established.

- WES plays a role in breaking the clinical tenet of explaining a complex disorder by a monogenic inheritance.

\section{Novel Insights}

- This study contributes to the understanding of blended phenotypes in the era of comprehensive molecular genotyping.

- The relevance of deep phenotyping to allow gene prioritization is essential.

- Is it just expanding the phenotype of a monogenic disorder or dual molecular diagnosis impacting clinical manifestations?

- DVL1-associated autosomal dominant Robinow syndrome exibits a severe phenotype due to synergistic effect of a BMP2 mutation - a possibility of a common interactive pathway.

\section{Keywords}

Blended phenotype $\cdot B M P 2 \cdot D V L 1 \cdot$ Reanalysis $\cdot$ Wholeexome sequencing

\section{Abstract \\ We report a family with a spectrum of short stature, cranio- facial dysmorphism, and digital anomalies in a father and 2 daughters, with the youngest (proband) displaying a severe phenotype. Clinically, autosomal dominant Robinow syn-}

\section{KARGER}

(C) 2020 S. Karger AG, Basel drome (ADRS) was diagnosed. Whole-exome sequencing identified a heterozygous pathogenic BMP2 variant in the father and his daughters. The phenotype of short stature, facial dysmorphism, and skeletal anomalies with or without cardiac anomalies related to BMP2 haploinsufficiency has some facial and digital resemblance to ADRS. Although this variant segregated in the affected members, it failed to explain the severe phenotype of the proband. A reanalysis of the girl's raw data confirmed 2 disorders: a de novo likely pathogenic DVL1 variant implicated in ADRS and the familial
Dr. Ratna Dua Puri

Institute of Medical Genetics and Genomics Sir Ganga Ram Hospital

Rajinder Nagar, New Delhi 110060 (India)

E-Mail ratnadpuri@yahoo.com 
$B M P 2$ variant. A close interplay of high-throughput sequencing and deep phenotyping unraveled the complexities of the blended phenotype in the proband.

(c) 2020 S. Karger AG, Basel

Robinow syndrome is a rare congenital skeletal dysplasia with dysmorphism characterized by a variable combination of short stature, mesomelia, genital hypoplasia, and distinctive fetal facies [Robinow et al., 1969]. It exhibits both autosomal dominant and recessive forms of inheritance. Mutations in genes typically encoding components of the non-canonical Wnt signaling pathway are implicated in this disorder, including ROR2 (MIM 602337) for autosomal recessive Robinow syndrome and WNT5A (MIM 164975), DVL1 (MIM 601365), and DVL3 (MIM 601368) for autosomal dominant Robinow syndrome (ADRS).

Herein, we report a family with 2 unrelated skeletal disorders segregating in different symptomatic members. We show the impact of variation in 2 genes accounting for a blended phenotype. We further discuss the role of next-generation sequencing (NGS) technology in characterizing the familial syndromes.

\section{Clinical Report}

The proband, a 3.9-year-old girl, second born to nonconsanguineous parents, presented with short stature and dysmorphic features. She had a history of delayed motor milestones, but normal cognitive development and no feeding problems. Her height was $71.8 \mathrm{~cm}$ (-6.2 SD), weight was $7.8 \mathrm{~kg}(-3 \mathrm{SD})$, and her head circumference was $52 \mathrm{~cm}(+2.4 \mathrm{SD})$. The upper segment/lower segment ratio was 1.5:1. Macrocephaly with wide-open fontanelle and frontal bossing was found. Dysmorphic features included hypertelorism, downslanting prominent eyes, infraorbital crease with midface hypoplasia, flat nasal bridge, short nose, anteverted nares, supernumerary teeth, dental crowding, gum hyperplasia, higharched palate, and a short neck. Downturned corners of the mouth and low-set ears were also observed (Fig. 1a, b). The salient skeletal abnormalities were mesomelia in both upper and lower limbs, bilateral broad great toes, brachydactyly of the 3rd and 4th fingers and all toes (Fig. 1c, d). The systemic examination was normal. Radiographs of the feet showed a bilateral absence of the middle phalanges in the 2nd-5th toes, bilateral absent distal phalanges in the 3 rd toes, and bilateral hypoplastic distal phalanges in the 1st, 2nd, 4th, and 5th toes (Fig. 1e).

Her elder sister had short stature $(119.5 \mathrm{~cm},-2.2 \mathrm{SD})$ with gingival hyperplasia, dental crowding, and bilateral clinodactyly (Fig. 2a). Radiographs of the feet showed bilateral hypoplastic distal phalanges. Bilateral big toes showed a broad base of the distal phalanges and short, broad proximal phalanges (Fig. 2b). The girl's father had short stature $(155.6 \mathrm{~cm},-2.77 \mathrm{SD})$, dental crowding, and short 3rd toes (Fig. 2c, d). Radiographs of the feet showed bilater- ally absent middle phalanges of the $3 \mathrm{rd}$, 4 th, and 5 th toes. Bilateral big toes showed a broad base of the distal phalanges as well as short and broad proximal phalanges (Fig. 2e). The mother was of average height and had no dysmorphism. The clinical evaluation of this family revealed a spectrum of short stature, a recognizable craniofacial gestalt, dental crowding, and digit anomalies except for the mother. A clinical diagnosis of ADRS was made with the proband displaying severe dysmorphism and the sister and father with a milder phenotype.

\section{Materials and Methods}

Whole-exome sequencing (WES) of the siblings and parents was performed after extracting the genomic DNA from peripheral venous blood samples. Extracted DNA was used to perform targeted gene capture using the Exome Research Panel (Integrated DNA technologies). The libraries were sequenced to mean $>80$ $100 \times$ coverage on the Illumina sequencing platform. The sequences obtained were aligned to the human reference genome (GRCh37/ hg19) using the BWA program [Li and Durbin, 2010; Meyer et al., 2013] and analyzed using Picard and GATK version 3.6 [Li et al., 2009; McKenna et al., 2010] to identify variants relevant to the clinical indication. Gene annotation of the variants was performed using the VEP program [McLaren et al., 2010] against the Ensembl release 87 . Clinically relevant mutations were annotated using published variants in literature and a set of disease databases ClinVar, OMIM, and HGMD (Human Gene Mutation Database). Common variants were filtered, based on allele frequency in 1000 Genome Phase 3, ExAC (Exome Aggregation Consortium), EVS (exome variant server), dbSNP147, 1000 Japanese Genome, and our internal Indian population database. Nonsynonymous variants effect was calculated using multiple algorithms such as PolyPhen-2, SIFT, MutationTaster2, and Mutation Assessor.

\section{Results}

WES identified a heterozygous, novel nonsense variant (c.508C $>\mathrm{T})$ in exon 3 of the BMP2 gene (chr20: $6759053 \mathrm{C}>\mathrm{T}$; depth: $31 \times$ ) that results in a stop codon and premature truncation of the protein at codon 170 (p.Arg170Ter; ENST00000378827) in the proband, her sister and their father, but not in the mother. We used standard variant filtration criteria such as common population variants based on allele frequency $>1 \%$, low-depth regions $(<20 \times)$, low quality score, and deep intronic variants. We found the BMP2 gene, one of the phenotypically associated genes as a loss-of-function allele in $B M P 2$ (PVS1), is implicated in craniofacial and skeletal features [Tan et al., 2017]. This variant is not described in the GnomAD (V2) database (PM2) and has pathogenic predictions from DANN, EIGEN, FATHMM-MKL, and MutationTaster 2 computational software with no benign prediction made by any software (PP3). Based on the in 

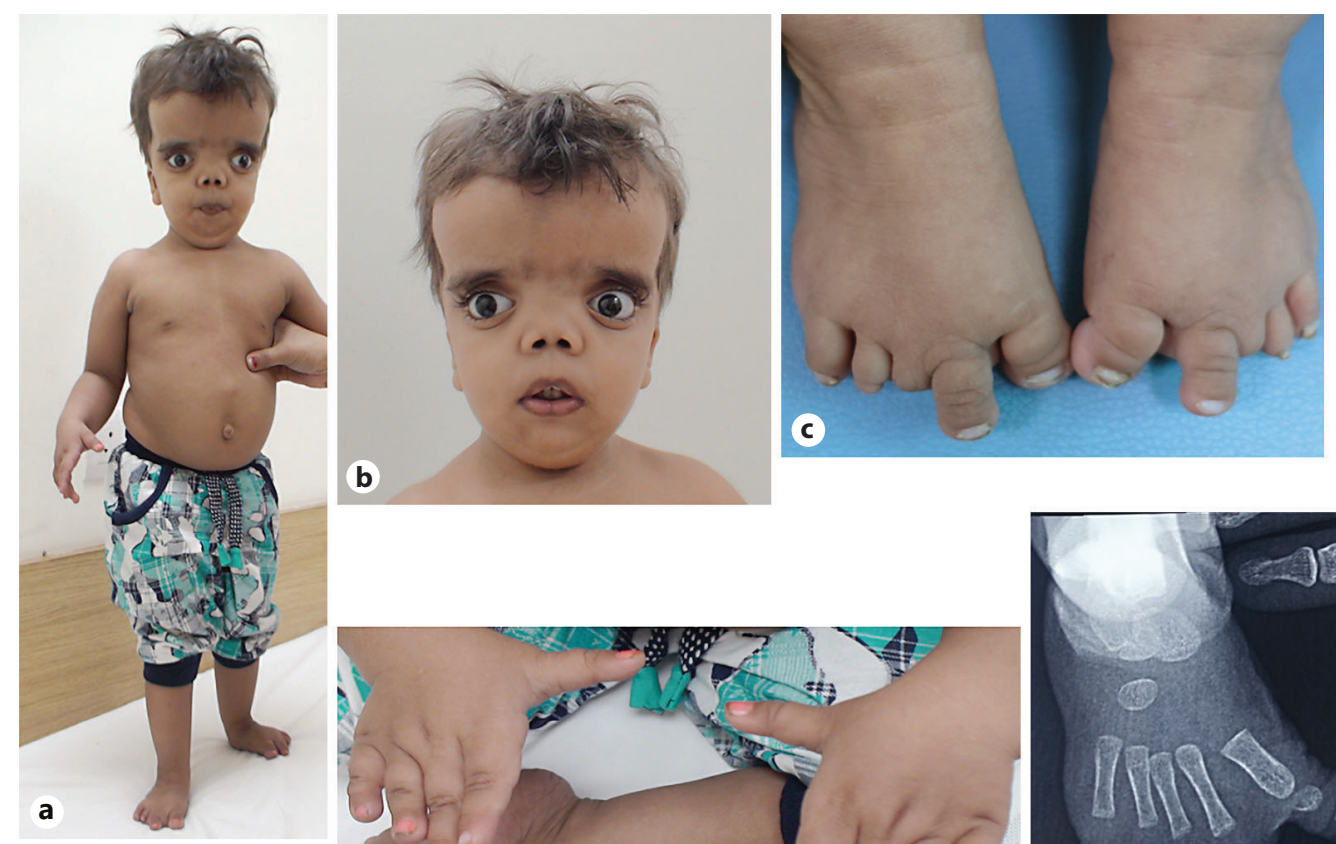

b
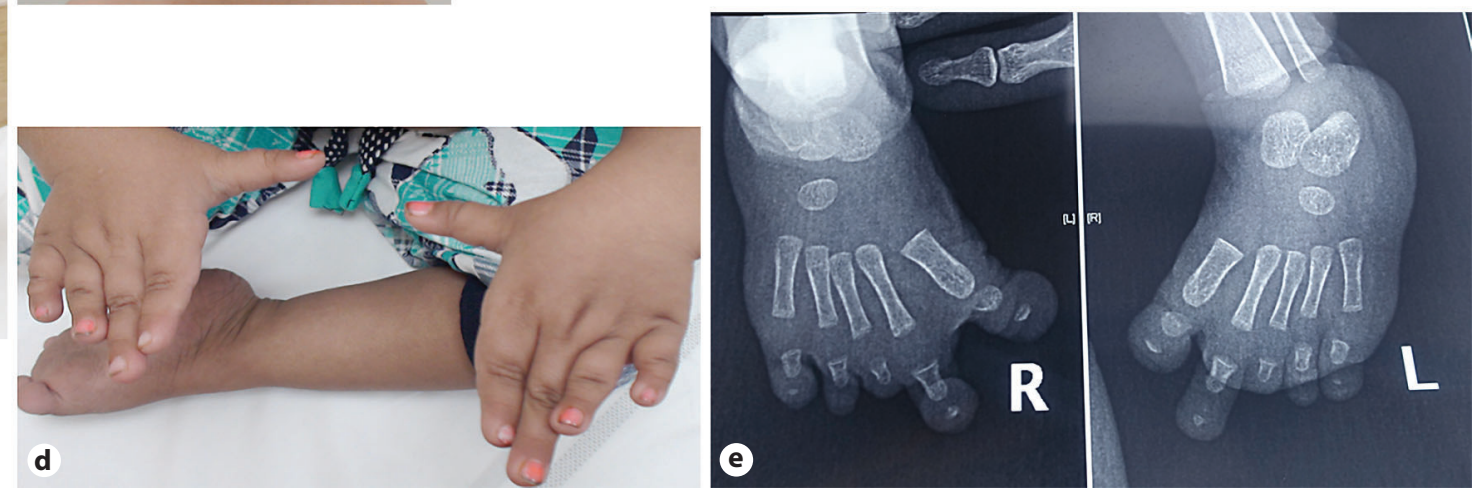

Fig. 1. a-e Photographs of the proband showing short stature, mesomelia (a), macrocephaly, hypertelorism, anteverted nares, dental crowding, short neck (b), sandal toe gap, brachydactyly of all toes (c), brachydactyly of third and fourth fingers, proximally placed thumbs (d). X-rays showing bilaterally absent middle phalanges of 2 nd -5 th toes, absent distal phalanx in 3 rd toe as well as hypoplastic distal phalanges in the 1 st, $2 \mathrm{nd}$, 4 th, and 5 th toes $(\mathbf{e})$.

silico predictions and the nature of the variant, it was classified as pathogenic against the American College of Medical Genetics and Genomics (ACMG) Standards and Guidelines [Richards et al., 2015]. While short stature and craniofacial, skeletal and dental features of the 3 members could be explained by the BMP2-related phenotype [short stature, facial dysmorphism, and skeletal anomalies with or without cardiac anomalies (SSFSC; MIM 617877)], the severity of the proband's dysmorphism was out of proportion. Hence, the genomic dataset was reanalyzed to search for Robinow or related syndromes, and the proband was found to carry a heterozygous likely pathogenic 13-bp de novo frameshift deletion in the DVL1 gene [ENST00000378891:c.1619_1631del; p.Thr540Arg fsTer105] causative of ADRS. This was absent in the elder sibling and the parents. ClinVar reports this frameshift deletion variant as "likely pathogenic." Since there is no clinical information in ClinVar, we report this as a novel variant for DVL1-associated ADRS. This frameshift variant lies in a mutation cluster region (PM1) and is de novo in this family (PS2). It is not present in the GnomAD (V2) database (PM2) and a -1 frameshifting indel in DVL1 (PVS1) is reported as a disease-causing mechanism for ADRS [White et al., 2018]. Based on the abovementioned features of the identified variant, it is reclassified as a pathogenic variant as per the ACMG Standards and Guidelines [Richards et al., 2015]. Sanger validation of the familial BMP2 single nucleotide change and the de novo DVL1 frameshift deletion was performed on the affected members (Fig. 3).

\section{Discussion}

Robinow syndrome is a genetically heterogeneous disorder with a distinct facial gestalt. The phenotype becomes less characteristic with age, and therefore, the diagnosis may not be as obvious [White et al., 2015]. In the family reported above, the youngest child had the typical fetal facies, while the elder sibling and father had a milder 


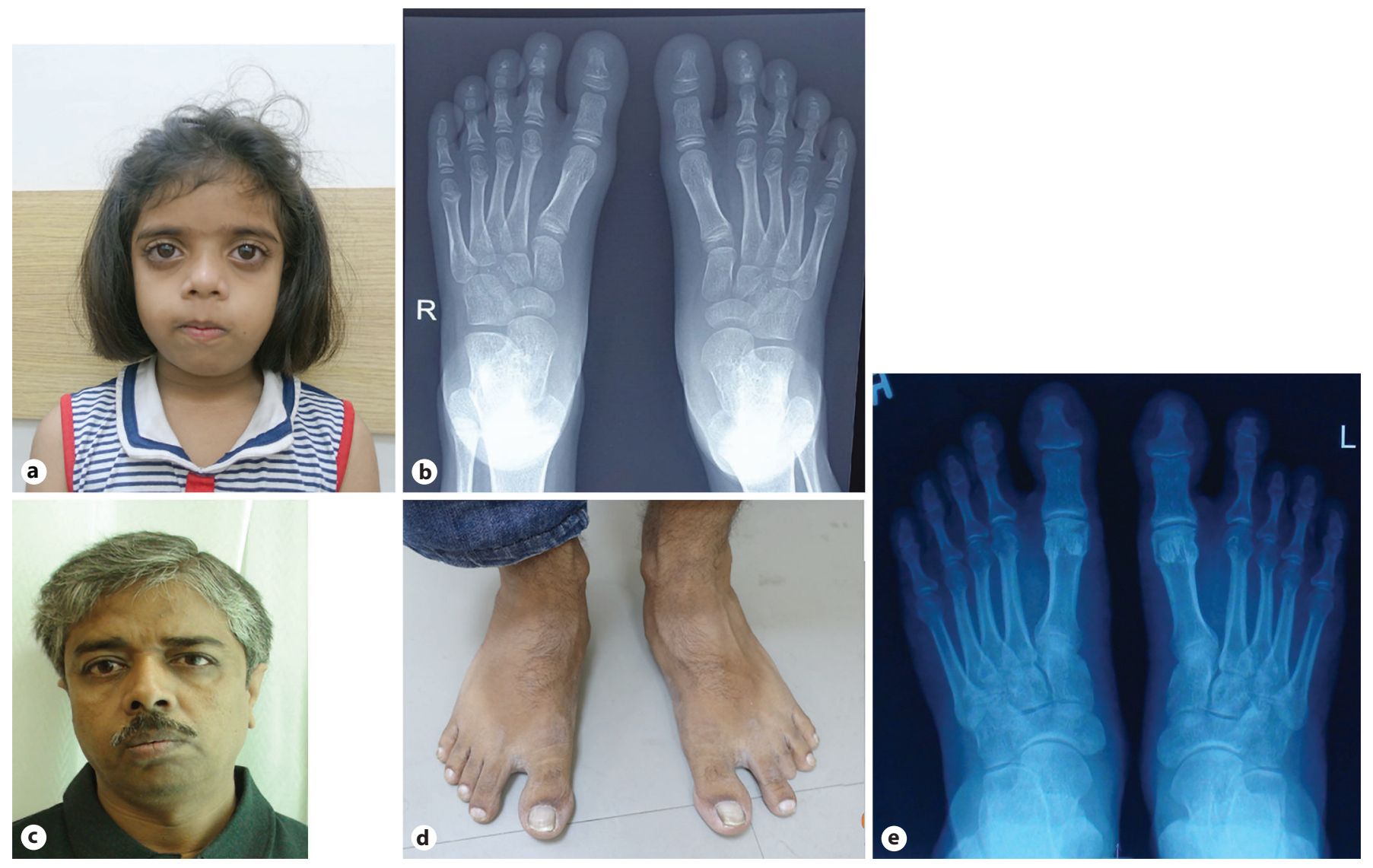

Fig. 2. a Proband's elder sibling. b X-ray of the sibling's foot showing hypoplastic distal phalanges. c Proband's father. d Father's feet showing sandal toe gap and short 3rd left toe. e Radiograph of the father's feet revealing absent middle phalanx of the 3 rd toe and hypoplastic distal phalanges of all toes.

phenotype, making their clinical diagnosis less obvious. DVL1-associated Robinow syndrome patients have significant macrocephaly (OFC $>+4 \mathrm{SD})$ and normal height [White et al., 2015]. However, these features were absent in our proband who had significant short stature at -6.2 SD and borderline macrocephaly at +2.48 SD.

Tan et al. [2017] have demonstrated that individuals heterozygous for $B M P 2$ truncating sequence variants or deletions display a consistent distinct phenotype characterized by short stature and skeletal features without neurological deficits. The clinical features of the 3 members from our family correlated with the SSFSC phenotype associated with $B M P 2$. While many of the craniofacial, skeletal, and dental features of the 8 individuals with BMP2 variants in the Tan et al. [2017] cohort were seen in our affected family members, the proband displayed an extended phenotype for BMP2 genotype (Table 1).
The possibility of a contiguous gene deletion syndrome was unlikely to explain the phenotype of the proband given the missense variation in the BMP2 gene. Expanding the phenotype of BMP2-related SSFSC syndrome or an additional monogenic disorder remained as possible explanations. To evaluate this, a reanalysis of the whole-exome raw data was done, and a heterozygous 13bp de novo exonic frameshift deletion in the penultimate exon 14 of the DVL1 gene was identified.

The era of NGS for monogenic disorders has brought about the concept of "blended phenotypes" that result from the interaction of 2 or more monogenic diseases in an individual [Posey et al., 2017]. This modifies the clinical phenotypes that traditionally were reported as extending phenotypes in established monogenic disorders. The youngest child in this family had significant facial dysmorphism that could not be attributed to the familial $B M P 2$ gene variant initially identified by exome sequenc- 


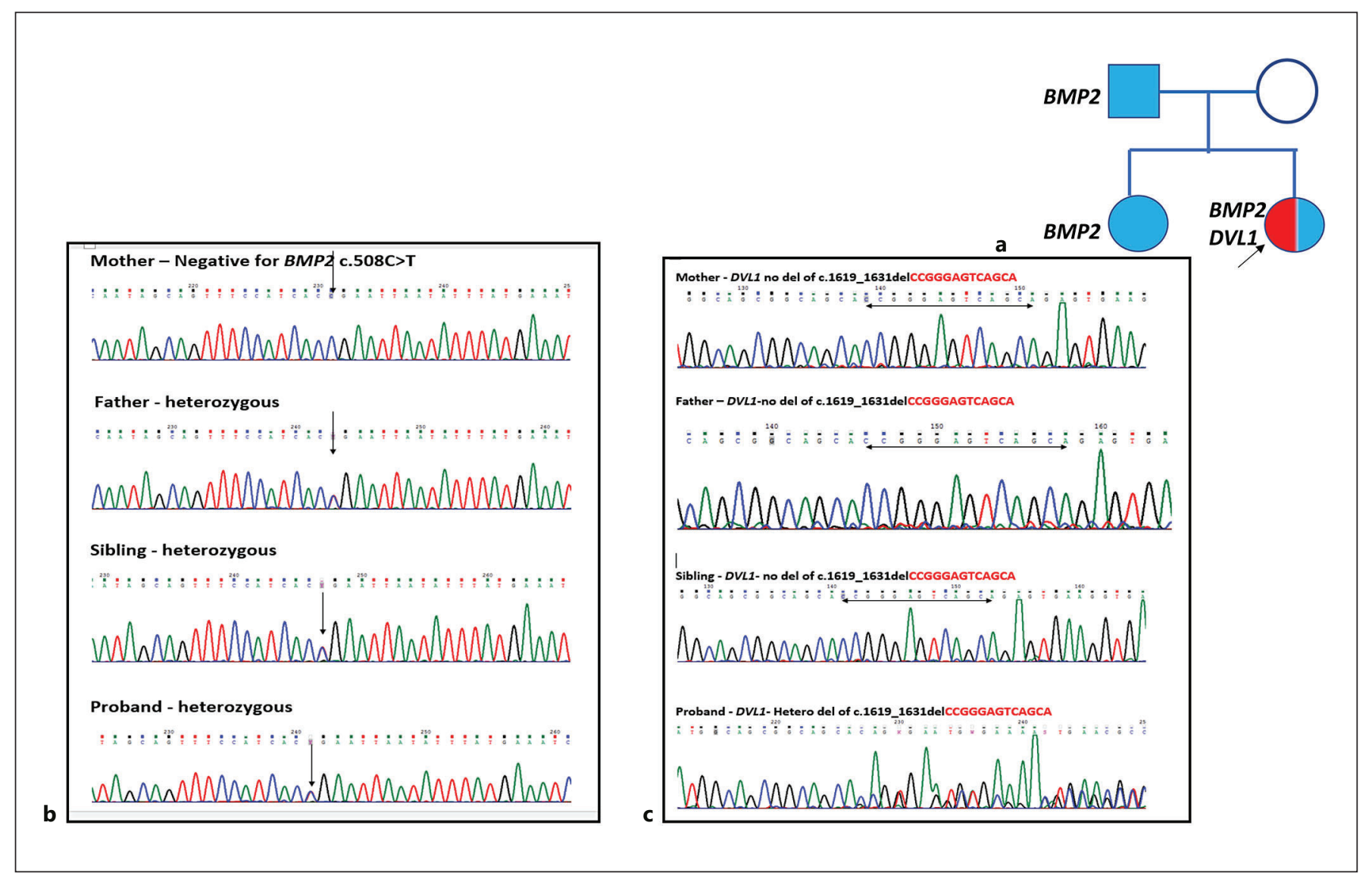

Fig. 3. a Pedigree of the family with the segregated variants. b Chromatograph of exon 3 of the BMP2 gene showing a heterozygous c.508C $>\mathrm{T}$ mutation in the proband, sibling and father. c Chromatograph of exon 14 of the DVL1 gene showing a heterozygous 13-bp frameshift deletion in the proband, but not in the sibling and the parents.

ing. We feel that overlapping pathways of $B M P 2$ and $D V L 1$ could be responsible for the blended phenotype as observed in the proband.

DVL1 encodes a segment polarity protein disheveled homolog DVL-1, which is an essential downstream mediator of the Wnt signaling pathway implicated in ADRS. Two types of Wnt signaling pathways have been characterized: the $\beta$-catenin-dependent canonical pathway and the $\beta$-catenin-independent non-canonical pathway; DVL1 mediates both signals [White et al., 2018]. So far, all variants in DVL1 associated with ADRS are identified in the penultimate or the last exon resulting in a -1 frameshift mutation which forms stable mRNA that escapes nonsense-mediated decay [Bunn et al., 2015; White et al., 2015, 2016, 2018]. The resultant mutant protein has a novel $\mathrm{C}$ terminal domain with a premature termination codon in exon 15. Bunn et al.
[2015] presented data to suggest that the mechanism of these alleles is indeed a gain of function that is contingent on the coexpression of both wild-type and mutant alleles.

Bone morphogenetic proteins (BMPs) are multifunctional growth factors which belong to the transforming growth factor $\beta$ (TGF- $\beta$ ) superfamily and exert critical actions during skeletal development [Liu et al., 2006]. Integration of BMP and Wnt signaling pathways occurs at several different molecular steps, including $\beta$-catenin and DVL. BMP promotes osteogenesis and may oppose proliferative pathways promoted by Wnt [Lin and Hankenson, 2011]. In their experimental models, Liu et al. [2006] have demonstrated that BMP-2 attenuates Wnt signaling through the formation of specific complexes between Dvl-1 and Smad1 that restricts $\beta$-catenin activation (canonical pathway) [Liu et al., 2006]. Hence, it can be pro- 
Table 1. Clinical characteristics of affected individuals with $B M P 2$ variants and deletions

\begin{tabular}{|c|c|c|c|c|}
\hline \multirow[t]{2}{*}{ Characteristics } & \multirow{2}{*}{$\begin{array}{l}\text { Tan et al., } 2017^{\mathrm{a}} \text {, } \\
n / N(\%)\end{array}$} & \multicolumn{3}{|l|}{ Present study } \\
\hline & & proband & sibling & father \\
\hline Height $\leq 2.0 \mathrm{SD}$ & $8 / 11(72.7)$ & $+(-6.22 \mathrm{SD})$ & $+(-2.2 \mathrm{SD})$ & $+(-2.77 \mathrm{SD})$ \\
\hline Broad forehead & $8 / 12(66.7)$ & + & - & - \\
\hline Temporal narrowing & $8 / 10(80.0)$ & - & - & - \\
\hline Synophrys & $5 / 9(55.6)$ & - & + & - \\
\hline Midface hypoplasia & $11 / 12(91.7)$ & + & - & + \\
\hline Low-set posteriorly rotated ears & $7 / 12(58.3)$ & + & + & + \\
\hline Short nose & $12 / 12(100)$ & + & + & - \\
\hline Anteverted nares & $12 / 12(100)$ & + & + & - \\
\hline Thin philtrum & $12 / 12(100)$ & + & + & + \\
\hline Palatal anomaly & $11 / 11(100)$ & - & - & - \\
\hline Dental crowding & $6 / 10(60.0)$ & + & + & + \\
\hline Anterior open bite & $5 / 10(50.0)$ & + & - & - \\
\hline 11 pairs of ribs & $6 / 9(66.7)$ & ND & $\mathrm{ND}$ & $\mathrm{ND}$ \\
\hline $\begin{array}{l}\text { Phalangeal abnormalities (mainly } \\
\text { clinodactyly) }\end{array}$ & $10 / 10(100)$ & $\begin{array}{l}\text { Hypoplasia to aplasia of } \\
\text { multiple phalanges }\end{array}$ & $\begin{array}{l}\text { Hypoplasia of distal } \\
\text { phalanges of all toes }\end{array}$ & $\begin{array}{l}\text { Aplasia of bilateral } \\
\text { middle phalanges of } \\
\text { 3rd-5th toes }\end{array}$ \\
\hline Sandal gap & 8/11 (72.7) & + & + & + \\
\hline Normal intellect & $11 / 11(100)$ & + & + & + \\
\hline Normal bone biochemistry & $8 / 8(100)$ & ND & ND & ND \\
\hline
\end{tabular}
cohort.

ND, not done. ${ }^{a}$ Only those features were compared which were present in 50\% or more individuals in the Tan et al., 2017

posed that the $B M P 2$ and $D V L 1$ variants had a synergistic effect in modifying the bone biology of the proband via the common $\beta$-catenin pathway. The sum of the disorders of the 2 pathways resulted in a more pronounced and atypical manifestation of ADRS in the proband in terms of significant short stature and extensive digital anomalies.

Indel mutation detection can be challenging in highthroughput sequencing data because of various errors associated with indel variant calling. To reduce the burden of false positives and false negatives, picking up an appropriate variant caller for indel detection becomes a necessity. This has been previously reported by White et al. [2015] and reiterates the need for reanalysis of the raw data at the DVL1 locus in patients with a strong suspicion of ADRS and an unyielding WES result [White et al., 2015]. As frameshift indels in the penultimate and last exons of DVL1 are the predominant mutations [Bunn et al., 2015; White et al., 2015, 2016], the variant caller has to be scripted for picking up these indels in the NGS data. This would go a long way in simplifying the diagnostic algorithm for such cases and facilitate cost-cutting by avoiding expensive Sanger sequencing as the first step in this algorithm.

\section{Conclusion}

This case highlights the importance of deep phenotyping in the era of high-throughput sequencing. It also elucidates the potential of advanced molecular technologies to identify and define blended phenotypes in patients with overlapping clinical features.

\section{Acknowledgment}

We would like to thank Dr. Thenral S Geetha for the NGS analysis in this case. The study was part of the Familial Genetic Disease Consortium (FGDS) sequencing effort.

\section{Statement of Ethics}

This study has been approved by the Institutional Ethics Committee. Informed consent was obtained from the parents for publication of this case report and accompanying images as well as molecular analysis.
48

Mol Syndromol 2020;11:43-49 DOI: $10.1159 / 000505506$
Mishra/Jain/Gupta/Saxena/Kulshreshtha/ Ramprasad/Verma/Dua Puri 


\section{Disclosure Statement}

The authors have no conflicts of interest to declare.

\section{Funding Sources}

This study was supported by the SciGenom Research Foundation (SGRF), Cochin, India.

\section{Author Contributions}

R. Mishra designed the study and prepared the initial draft of the manuscript. V. Jain did the clinical evaluation of the case. D. Gupta performed Sanger sequencing of the identified variants. R. Saxena supervised Sanger sequencing and the analysis of the identified variants. S. Kulshreshtha did NGS reanalysis and V.L. Ramprasad supervised the initial WES of the family. I.C. Verma was in charge of overall patient management, preparation, and finalization of the manuscript providing important intellectual inputs. $\mathrm{R}$. Dua Puri made the diagnosis, supervised patient work-up and management, revised and finalized the manuscript.

\section{References}

Bunn KJ, Daniel P, Rösken HS, O’Neill AC, Cameron-Christie SR, et al: Mutations in DVL1 cause an osteosclerotic form of Robinow syndrome. Am J Hum Genet 96:623-630 (2015).

Li H, Durbin R: Fast and accurate long-read alignment with Burrows-Wheeler transform. Bioinformatics 26:589-595 (2010).

- Li H, Handsaker B, Wysoker A, Fennell T, Ruan J, et al: The sequence alignment/map format and SAMtools. Bioinformatics 25:2078-2079 (2009).

Lin GL, Hankenson KD: Integration of BMP, Wnt, and notch signaling pathways in osteoblast differentiation. J Cell Biochem 112: 3491-3501 (2011).

Liu Z, Tang Y, Qiu T, Cao X, Clemens TL: A dishevelled-1/Smad1 interaction couples WNT and bone morphogenetic protein signaling pathways in uncommitted bone marrow stromal cells. J Biol Chem 281:17156-17163 (2006).

-McKenna A, Hanna M, Banks E, Sivachenko A, Cibulskis K, et al: The Genome Analysis Toolkit: a MapReduce framework for analyzing next-generation DNA sequencing data. Genome Res 20:1297-1303 (2010).
McLaren W, Pritchard B, Rios D, Chen Y, Flicek $\mathrm{P}$, et al: Deriving the consequences of genomic variants with the Ensembl API and SNP Effect Predictor. Bioinforma Oxf Engl 26:20692070 (2010).

Meyer LR, Zweig AS, Hinrichs AS, Karolchik D, Kuhn RM, et al: The UCSC Genome Browser database: extensions and updates 2013. Nucleic Acids Res 41:D64-D69 (2013).

Posey JE, Harel T, Liu P, Rosenfeld JA, James RA, et al: Resolution of disease phenotypes resulting from multilocus genomic variation. $\mathrm{N}$ Engl J Med 376:21-31 (2017).

Richards S, Aziz N, Bale S, Bick D, Das S, et al: ACMG Laboratory Quality Assurance Committee. Standards and guidelines for the interpretation of sequence variants: a joint consensus recommendation of the American College of Medical Genetics and Genomics and the Association for Molecular Pathology. Genet Med 17:405-424 (2015).
Robinow M, Silverman FN, Smith HD: A newly recognized dwarfing syndrome. Am J Dis Child 117:645-651 (1969).

Tan TY, Gonzaga-Jauregui C, Bhoj EJ, Strauss $\mathrm{KA}$, Brigatti K, et al: Monoallelic BMP2 variants predicted to result in haploinsufficiency cause craniofacial, skeletal, and cardiac features overlapping those of $20 \mathrm{p} 12$ deletions. Am J Hum Genet 101:985-994 (2017).

White J, Mazzeu JF, Hoischen A, Jhangiani SN, Gambin T, et al: DVL1 frameshift mutations clustering in the penultimate exon cause autosomal-dominant Robinow syndrome. Am J Hum Genet 96:612-622 (2015).

White JJ, Mazzeu JF, Hoischen A, Bayram Y, Withers $\mathrm{M}$, et al: $D V L 3$ alleles resulting in a -1 frameshift of the last exon mediate autosomal-dominant Robinow syndrome. Am J Hum Genet 98:553-561 (2016).

White JJ, Mazzeu JF, Coban-Akdemir Z, Bayram $\mathrm{Y}$, Bahrambeigi V, et al: WNT signaling perturbations underlie the genetic heterogeneity of Robinow syndrome. Am J Hum Genet 102: 27-43 (2018). 